\title{
Rebutting Pedro J. Silva's article in Biophysical Chemistry supporting Mitchell-Boyer's ideas on bioenergetics and advocating murburn concept as a viable explanation for aerobic respiration and oxygenic photosynthesis
}

(Letter to the Editor)

by

Kelath Murali Manoj ${ }^{*}$

* Corresponding author, Satyamjayatu: The Science \& Ethics Foundation,
Snehatheeram, Kulappully, Shoranur-2 (PO), Kerala, India-679122.
murman@ satyamjayatu.com

Abstract: Over the last three years, I had pointed out the untenable nature of the proton-centric 'chemiosmosis driven rotary ATP-synthesis (CRAS)' explanation for Oxidative Phosphorylation (OxPhos). Recently, Pedro J. Silva (PJS) [Chemiosmotic misunderstandings (2020). Biophys. Chem. 264, 106424] afforded a part of our work his critical attention, but overlooked the large volume of evidence against CRAS and supporting the oxygen-centric murburn mechanism of OxPhos. In his article, PJS also posed some queries on our bioenergetics model. When I offered my rebuttal, the Editor of Biophysical Chemistry refused to publish it. Therefore, I have no other option than to publish the rebuttal as a preprint. Herein, I demonstrate the flaws and lacunae in PJS's defense of CRAS hypothesis and answer his specific queries and defend the murburn explanation of mOxPhos. The current scientific discourse is crucial for correcting major historical errors in mitochondrial physiology and understanding oxygen's crucial role in the powering chemistry of life.

Keywords: bioenergetics; chemiosmosis; proton-motive force; murburn concept; aerobic respiration; mitochondria; oxidative phosphorylation; 
This letter pertains to Pedro J. Silva's (PJS) recent paper supporting the chemiosmostic rotary ATP synthesis (CRAS) hypothesis [1] and his queries/critique on Manoj's murburn explanation for mitochondrial oxidative phosphorylation (mOxPhos) [2-18]. Before I get to the crux, I would like to express a note of reservation at the inappropriate attributions made to our writing/pursuit and Biophysical Chemistry's editorial's reluctance to note these issues. In his maiden research publication on mOxPhos, PJS postures authoritatively at the outset when he calls our work 'controversial' and pacifies the community to maintain a 'status quo' in the field and not look for new answers. Nikolai Bazhin, my fellow crusader in the anti-CRAS pursuits [7,16,19-21], is an expert with more than six decades' research experience in biochemical thermodynamics and kinetics. Several renowned pioneers of the past like- Edward Slater, Robert Williams, Britton Chance, Gilbert Ling, David Green, Albert Lehninger, Walter Wainio, Gregorio Weber, etc. had vehemently critiqued CRAS. Over the decades, several others had floated parallel explanations for OxPhos, even after Peter Mitchell \& Paul Boyer were recognized with Nobel Prizes. These realities indicate that the CRAS explanation is inadequate and even PJS himself admits in the end of his article that- "It (chemiomsosis) may eventually be found wanting, but parsimony and intellectual humility should force us to postpone its replacement to a moment when enough shortcomings have been found and a novel paradigm which offers as much explanatory power is ready". If everything was explicable with the CRAS mechanism, why should such a statement arise? One wonders if pursuits in science and repositories of truth are entitled to only certain 'chosen' individuals to challenge and to establish.

I must stress that it is an honorable and egalitarian tradition in scientific pursuits to differ and debate; and there is nothing controversial in a scientist presenting a new evidence-based mechanistic perspective. It would have served everyone's cause if PJS's critique was tempered with a greater awareness of the lacunae in the field, which we and others had exposed [Supplementary File, Item 1; for editorial/peer review]. In our writings, I had disclaimed clearly that we dealt with the consensus data/ideas within textbooks (Lehninger/Stryer/Voet) and reviews, and this was duly cited at the outset of the two articles from my group that PJS cites [Supplementary File, Item 2; for editorial/peer review]. PJS writes that we did not give sources for the data we critiqued on the stoichiometry of proton pumps and the text that we cited for the binding constants of Complex V (for ADP/ATP) lacked this information. The fact relevant to the 
discussion was that the various Complexes' reaction $\Delta \mathrm{G}$ values could not afford the number of protons pumped (which was either 2 or 4; and we had inclusively dealt with both scenarios) [3,6]. With respect to Complex V's affinity being higher for ATP (in comparison to ADP, thereby making it an ATPase!), the cited textbook has the specific data we perused [Supplementary File, Item 3; for editorial/peer review]. I quote verbatim select contents of a section titled "ATP is stabilized relative to ADP on surface of $F_{1}$ " from the revered textbook, Lehninger: Principles of Biochemistry (Chapter 19, page 709, left column, paragraph 3) that many of us have perused for long, as professors and professionals of Biochemistry-

"ATP synthase stabilizes ATP relative to $A D P+P i$ by binding ATP more tightly, releasing enough energy to counterbalance the cost of making ATP. Careful measurements of the binding constants show that FoF1 binds ATP with very high affinity $\left(K_{d} \leq 10^{-12} \mathrm{M}\right)$ and $A D P$ with much lower affinity $\left(K_{d} \approx 10^{-5} \mathrm{M}\right)$."

The crux of the matter is that in both cases, there was nothing wrong with the data we perused/presented as we were inclusive regarding the literature on the field. Rather than trying to project that our publications were "carelessly written by an amateur", PJS could have addressed how- (i) the reaction energetics as given in textbooks could be justified for 2/4 proton pumped by the Complexes and (ii) an enzyme that has more than ten million fold higher affinities for ATP over ADP can serve as the physiological ATP-synthase in mitochondria, wherein [ATP] exceeds [ADP]. Further, when PJS says that I consider 'ETC to be a source of DROS', it is gross injustice to our works because we do not consider that there is any such thing as ETC itself [3,6-8,14]. Very importantly, sidelining our reproduced experimental work (demonstration of DROS mediated ATP synthesis from ADP and Pi, the most crucial evidence to our theory $[6,15]$ ) as artifact is an unacceptable approach at PJS's end. Rather than wishing it away, if he did the experiment and refuted my group's findings, it would have made more sense and purpose. Now, the attention focuses on the crux of the scientific discourse, which I present in three heads.

(I) Firstly, I argue that the chemiosmosis paradigm has been demonstrated to be a false concept from an $a b$ initio perspective, as there are little protons within mitochondrial matrix to serve the purported proton pumps [2-4,6-8]. Further, the steady-state workability concerning ETC-CRAS hypothesis is given in a new schematic perspective, within Figure 1. A dynamic working model of a polarized electrical system allows only directional flow of positive or negative charges (topright) across partitions. However, the ETC-CRAS scheme seeks a deterministic 'flow/transfer' of one or two electrons against gradients and against polarities (top-left) in a very deterministic and 
serial fashion, often involving multi-molecular complexes, flouting known thermodynamics and not meeting the kinetic requirements to explain the overall catalytic rates. This is when neither a push nor a pull is deemed operative in the system. It is unacceptable that $\mathrm{O}_{2}$ has no role other staying bound at Complex IV to make water. Seen in a mechanical purview, pumps work directionally in an open system and the flow can be used only in a disconnected phase or the projected particles must conserve kinetic energy. However, the ETC-CRAS proposal is 'unworkable', as the recycling of protons is within the closed purview of IMS and matrix phases, across the very same inner mitochondrial membrane (IMM) (bottom-left). Protons can be pumped via the outer mitochondrial membrane (OMM) into the inter-membrane space (IMS) phase and they could be harnessed when entering the matrix phase via the purported motor of Complex V (bottom-right). Therefore, the electro-mechanical ETC-CRAS model of ATP synthesis is untenable. Further details of the critical dissection of the concepts of ETC, proton pumps, chemiosmosis and rotary ATP synthesis are available elsewhere [2-8].

(II) Next, I dissect PJS's 'new calculations supporting chemiosmosis' on square logical grounds, and in the light of our works. PJS serves the same old wine re-brewed in a new bottle, as his work is built on the modified Mitchell's proton motive force proposal and RAS model's unbalanced ATP synthesis equations (equation no. 1 and equation no. 12/13 respectively, of PJS's paper). Equation 1 has no grounding in reality or thermodynamics [7]. Further, the unbalanced equation $12 / 13$ is inappropriate to account for explaining the coupling of NADH oxidation with steady-state ATP synthesis within mitochondria [6,7]. Further, there is no way equation 1 can couple with the equations $12 / 13$ in reality and therefore, there is no real explanation afforded by ETC-CRAS. There is nothing called 'proton motive force (pmf)' outside the realm of bioenergetics and it exists in this field only because of faulty assumptions and misplaced observations taken as experimental supports. (If we permit the relation of $2=3$, it takes only a couple of steps to prove the arbitrary; zero equals infinity or $\pi$ or 1005 ! If we accept erroneous proposals as facts, then we need to entertain vain pursuits that lead us nowhere.) Outward pumping of non-existing protons by non-existing pumps cannot magically conserve energy on the projected IMS protons. Further, there is no electro-mechanical way to use the protons for ATP-synthesis using an inward flow across the same membrane. In simulated systems, when external proton gradients are given, ATP synthesis is facilitated due to an 
equilibrium-driven reaction, which cannot be operative in mitochondrial physiology. Most physiological bioenergetic systems are known to work with little $\mathrm{pH}$ gradient, whereas Mitchell's proposal originally sought large $\mathrm{pH}$ gradients of several units across the inner mitochondrial membrane. Accounting for the lack of an observable large $\mathrm{pH}$ gradient by conveniently adding a large electrical term is unjustifiable, as it creates energy from nowhere! The mitochondria are proton deficient systems that work with oxygen-centered radicals and ions, and the reaction needs protons for neutralization, to form $\mathrm{O}-\mathrm{H}$ bonds. This is particularly the case when starting with NADH, as it gives two electrons but only one proton. The way life uses this molecule is a wonderful thermodynamic feat. Protons contribute a large $\Delta_{\mathrm{f}} G^{\prime \mathrm{o}}$ aq of $452 \mathrm{~kJ} / \mathrm{mol}$ and this was overlooked by all in the field [16]. So, when NADH gets oxidized and DROS form and they attack $\mathrm{ATP}+\mathrm{Pi}$, the formation of $\mathrm{O}-\mathrm{H}$ bonds in peroxide, water, quinols, etc. drive the equilibrium to the right. This is the physiological coupling reaction. Even in the simulated equilibrium-driven ATP-synthesis by Complex V (ADP $\left.+\mathrm{Pi}+\mathrm{H}^{+} \rightarrow \mathrm{ATP}+\mathrm{H}_{2} \mathrm{O}\right)$ in vesicles, the reaction would need a cytoplasmic proton for effective ester bond formation within the closed/aprotic mitochondrial matrix. Therefore, providing a proton gradient (as in the famed Jagendorf's experiment) gives enhanced ATP formation, and this has no bearing with any 'pmf'.

(III) Finally, I present evidence and arguments supporting the murburn explanation of mOxPhos. It is the only standing rationale for cyanide toxicity towards several cellular physiologies $[11,12,15]$. A biochemical mechanism is ratified by its potency to explain inhibitions and the murburn theory also explains the outcomes of mitochondrial toxic principles of vitamin A, rotenone, oligomycin, etc. and a bevy of interfacial uncouplers [6,7] whereas Mitchellian proposal falls flat. The existence of ADP binding sites on various Complexes was demonstrated by in silico works and supported by experimental data from other workers [6]. The presence of various one electron redox active centers on the Complexes and their abilities to independently make DROS is direct support of the unordered murburn scheme of mOxPhos. The increase of DROS levels with provision of NADH/oxygen and the generation of higher TMP/ATP levels with concomitant increase in DROS are yet other potent physiological evidence. Theoretically, the murburn equations for OxPhos afford direct connectivity of NADH oxidation with ATP synthesis and the thermodynamic pull explains the substrate-dependent depletion of redox equivalents $[6,7,16]$. Most importantly, pmf cannot catalyze the synthesis of an ester bond in a 
foreseeable way, whereas DROS is capable mediating the formation of ester bond, theoretically and experimentally (in vitro and in situ). Till date, there is no observation reported that violates the premises of the murburn explanation for mOxPhos and we have listed 30 different issues where the murburn explanation trumps over the ETC-CRAS model [7] and PJS does not address this aspect at all. Our works with HPLC (employing elaborate controls) reproduced the results of the preliminary studies on DROS-assisted ATP synthesis and extended it to ratify the proposals with probes for cyanide inhibition [15]. Very importantly, we have quoted the experimental reports by various other pioneering researchers like Cohn/Drysdale, Galina Mironova, Tyszkiewicz/Roux, Kathleen Mailer, etc. to support our works, which confirm ROS-assisted ATP synthesis in mitochondria and chloroplasts [Supplementary Information, Item 4; for editorial review]. Furthermore, our DROS-based theory is solidly grounded in thermodynamics, kinetics and most known aspects of the mitochondrial reaction system and justifies the greater role and need for oxygen in aerobic life. PJS's arguments on the 'non-obligatorily required status' of Complex V in diverse single-celled organisms cannot address the high energy requirements and dynamic ATP turnovers for the organisms (we cited) to survive and grow under aerobic conditions. Further, our theory is also bolstered by the total absence of mitochondrial Complexes I, III \& IV (the purported proton pumps of Mitchellian theory) in the hypoxic growth conditions of the newly discovered cnidarian, Henneguya [21]. The fact that it has only Complex II \& V can be explained by murburn concept [16] but the Mitchell model fails.

We have explained clearly that the phosphorylation reaction must occur at the phospholipid interface to enhance the yields, and is furthered by a closure of the microcosm (lowering free protons to stabilize radicals and interfactial proton-dynamics) and the multiple one-electron redox centers within the membrane-embedded proteins are a testament to our theory [2-18]. We have also explained such facets in the relatively simpler heme proteins, like cytochrome P450 mediated catalysis and in the complex chloroplast systems [Supplementary Information, Item 5; for editorial review]. In fact, in our predictive work [7], we had projected the gradual increase in yields with the addition of various components of the reaction system. Further, while oxygencentered radicals and ions feature in aerobic systems, other ions/radicals (based in $\mathrm{N}, \mathrm{S}, \mathrm{Fe}$, etc.) could feature in the anaerobic/anoxic systems [7,16]. Also, enzymes such as nitrate reductase possess heme and FeS clusters and can work in a manner which is analogous to mitochondrial oxygen-dependent respiration. In this regard, our theory explains the higher efficacy, diversity 
and growth rates of oxygenic metabolic systems. Oxygen has direct equilibrium with water and gives $\mathrm{CO}_{2}$ as the primary product, which can be easily voided and it also provides facile homeostatic machine logic [7,16]. The one-electron route for synthesis of energy currency goes via the simple cycle of "oxygen - superoxide - peroxide - hydroxyl radical - hydroxide ion water", and it can be easily visualized under this view why addition of external protons/gradients enables enhanced ATP synthesis in physiological setups. Murburn paradigm is consistent with the employment of diverse metal centers in various metabolic and physiological routines of archaeobacteria, anaerobic micro-organisms and prokaryotes. We have also critiqued the ETCCRAS based explanations for oxygenic photophosphorylation and provided murburn concept based alternatives worth exploration [22-27]. The transition from various inorganic ionic/radical species in primitive microbes to oxygen-centered ionic/radical species is also chemically consistent with the murburn theory but inconsistent with purely affinity-based or structure-based electron transfers/proton pumps. While the ETC-CRAS explanation is definitely "irreducibly complex" (ref. Michael Behe), murburn theory is a simple and evolvable system of molecules, ions and radicals that can be envisaged to work spontaneously in heterogeneous or homogeneous phases. When PJS speaks of 'intellectual parsimony', I must say that it is murburn concept that fits the bill, not ETC-CRAS (Table 1). In toto, the murburn proposal provides a meaningful molecular mechanistic scaffold for the metabolism-based powering logic of life. It is currently the only theory that explains why oxygen is needed, how respiratory metabolism is connected to thermogenesis/homeostasis and why oxygen became the major elixir for life along with the solvent of water, and why the phosopholipid membrane became a vital cell-component [16].

\section{References (* Not peer reviewed, available freely as preprint with DOI)}

[1] P.J. Silva, Chemiosmotic misunderstandings, Biophys. Chem. 264 (2020) 106424. doi:10.1016/j.bpc.2020.106424.

[2] K.M. Manoj, Debunking chemiosmosis and proposing murburn concept as the explanation for cellular respiration, Biomed. Rev. 28 (2017) 35-52.

[3] K.M. Manoj, Aerobic respiration: criticism of the proton-centric explanation involving rotary ATP synthesis, chemiosmosis principle, proton pumps and electron transport chain, Biochem. Insights 11 (2018) 1178626418818442.

[4] K.M. Manoj, D.A. Gideon, V.D. Jacob, Murburn scheme for mitochondrial thermogenesis, Biomed. Rev. 29 (2018) 73-82.

[5] K.M. Manoj, The ubiquitous biochemical logic of murburn concept, Biomed. Rev. 29 (2018) 8997.

[6] K.M. Manoj, et al., Aerobic respiration: proof of concept for the murburn perspective, J. Biomol. 
Struct. Dyn. 37 (2019) 4524-4556.

[7] K.M. Manoj, et al., Chemiosmotic and murburn explanations for aerobic respiration: predictive capabilities, structure-function correlations and chemico-physical logic, Arch. Biochem. Biophys. 676 (2019) 108128.

[8] D.A. Gideon, V.D. Jacob, K.M. Manoj, 2020: Murburn concept heralds a new era in cellular bioenergetics, Biomed. Rev. 30 (2019) 89-98.

[9] Torday's prognosis for aging and mortality: more evolution and better life!, Biomed. Rev. 30 (2019), 23-24.

[10] V.D. Jacob, K.M. Manoj, Are adipocytes and ROS mistaken for villains, or are they protagonists in the drama of life? The murburn perspective, Adipobiology 10 (2019) 7-16.

[11] K.M. Manoj, V. Soman, Classical and murburn explanations for acute toxicity of cyanide in aerobic respiration: A personal perspective, Toxicology 432 (2020) 152369.

[12] K. Wallace, Challenging the current paradigm of the chemiosmotic theory for cyanide toxicity, Toxicology 432 (2020) 152377.

[13] K.M. Manoj, Refutation of the cation-centric torsional ATP synthesis model and advocating murburn scheme for mitochondrial oxidative phosphorylation, Biophys. Chem. 257 (2020) 106278.

[14] K.M. Manoj, Murburn concept: a paradigm shift in cellular metabolism and physiology, Biomol. Concepts 11 (2020) 7-22.

[15] K.M. Manoj, et al., Acute toxicity of cyanide in aerobic respiration: Theoretical and experimental support for murburn explanation, Biomol. Concepts 11 (2020) 32-56.

[16] K.M. Manoj, N.M. Bazhin, Murburn precepts of aerobic respiration. OSF Preprints (2019) doi: 10.31219/osf.io/hx4p9.

[17] K.M. Manoj, et al.*. What is the role of lipid membrane-embedded quinones in ATP-synthesis? Chemiosmotic Q-cycle versus murburn reaction perspective. OSF Preprints (2020). doi: 10.31219/osf.io/7zbax

[18] K.M. Manoj,* D.A. Gideon. Roles of cytochromes c and b5 in mitochondria and microsomes: classical and murburn perspectives." OSF Preprints (2020). doi:10.31219/osf.io/8a6ej.

[19] N.M. Bazhin, The essence of ATP coupling. ISRN Biochemistry 2012 (2012) 827604.

[20] N.M. Bazhin, Proton gradient energy in the catalytic ATP synthesis, React. Kinet. Catal. Lett. 90 (2018) 401.

[21] D. Yahalomi, et al., A cnidarian parasite of salmon (Myxozoa: Henneguya) lacks a mitochondrial genome. Proc. Natl. Acad. Sci. USA 117 (2020) 5358-5363.

[22] K.M. Manoj*, et al., Murburn precepts for the light reaction of oxygenic photosynthesis. OSF Preprints (2020). doi: 10.31219/osf.io/95brg.

[23] K.M. Manoj*. Critical analysis of some assumptions and observations on photolytic oxygenesis by plant cells. OSF Preprints (2020). doi: 10.31219/osf.io/y62j5

[24] D.A. Gideon, V. Nirusimhan, K.M. Manoj*. Are plastocyanin and ferredoxin specific electron carriers or generic redox capacitors? Classical and murburn perspectives on two chloroplast proteins. OSF Preprints (2020). doi: 10.31219/osf.io/j7q5v

[25] K.M. Manoj*, et al. Is Z-scheme a tenable explanation for the light reaction of oxygenic photosynthesis? OSF Preprints (2020). doi: 10.31219/osf.io/v6tdf

[26] K.M. Manoj*, et al. Microsomal xenobiotic metabolism, mitochondrial oxidative phosphorylation and cyanobacterial photorespiration have common murburn mechanistic underpinnings with chloroplastid photosynthetic physiology." OSF Preprints (2020). doi:10.31219/osf.io/8p2sx

[27] K.M. Manoj*, A. Manekkathodi. Light's interaction with pigments in chloroplasts: The murburn perspective. OSF Preprints (2020). doi: 10.31219/osf.io/wx4gv 
Figure \& Table

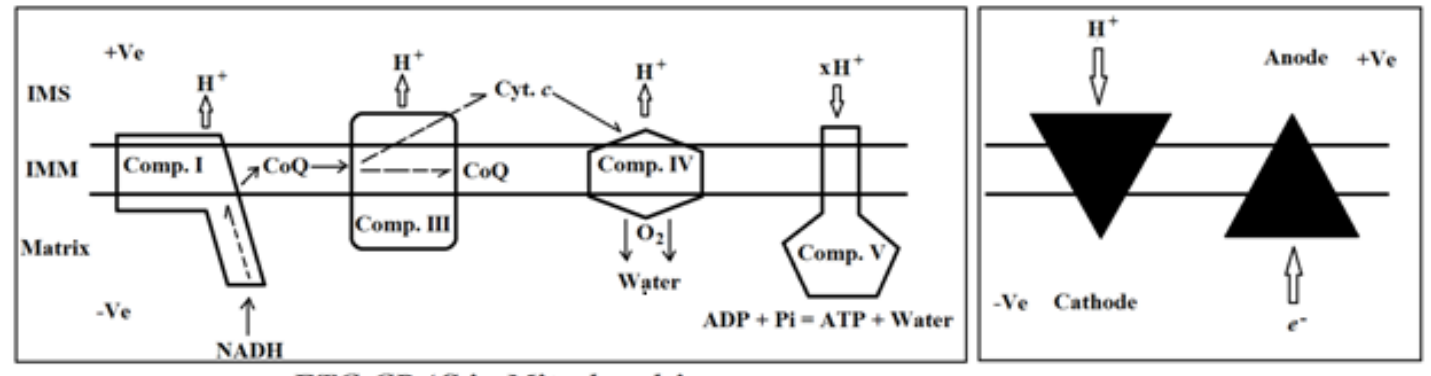

ETC-CRAS in Mitochondria

Workable electrochemical system

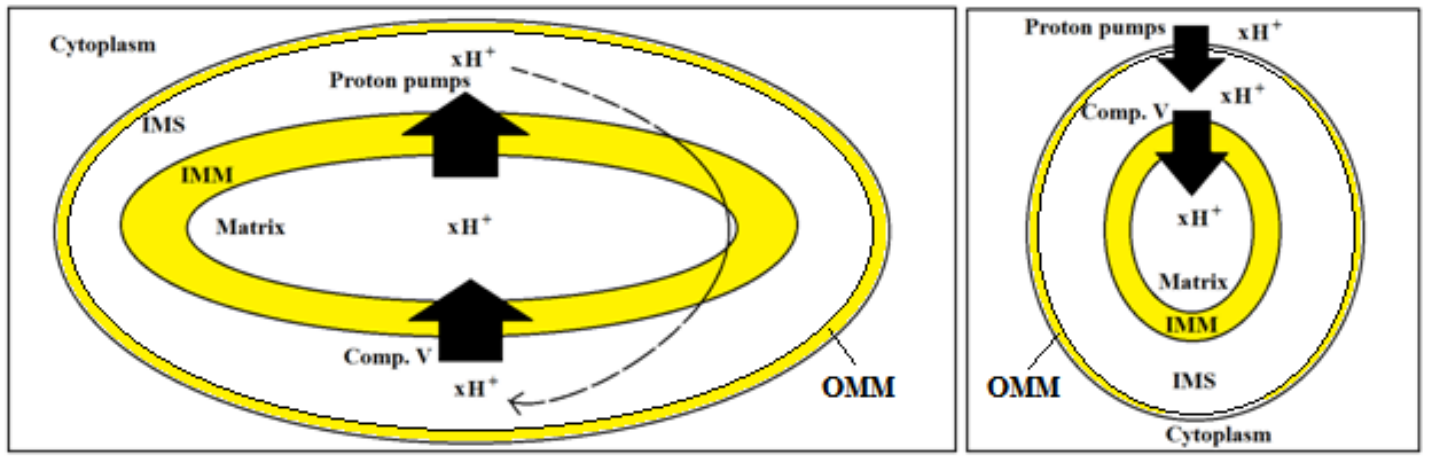

Proton motive force and rotary ATP synthesis

Workable pump-motor connectivity

Figure 1: A comparison of the electrochemical (top panels) and mechanical (bottom panels) purviews of the unrealistic ETC-CRAS proposal with realistic working models.

Table 1: A comparison of the ETC-Chemiosmosis-Rotary ATP synthesis and murburn paradigms for mitochondrial oxidative phosphorylation

\begin{tabular}{|c|c|c|c|}
\hline No. & Criteria & Murburn paradigm & ETC-CRAS model \\
\hline$\overline{1}$ & $\begin{array}{l}\text { What are the } \\
\text { minimal } \\
\text { reaction } \\
\text { components } \\
\text { required? } \\
\text { Does the } \\
\text { mechanism } \\
\text { qualify for } \\
\text { Michael } \\
\text { Behe's } \\
\text { "irreducible } \\
\text { complexity"? }\end{array}$ & $\begin{array}{l}\text { A molecule/ion serving as a source of electrons } \\
\text { (with low redox potential, like NADH or } \\
\text { sulfide) }+ \text { a } d \text { orbital/ } / \text {-electronic system to } \\
\text { serve as catalyst (like heme or flavin or Cu ion) } \\
+ \text { an acceptor molecule/ion of electrons (like } \mathrm{O}_{2} \\
\text { or Fe } \mathrm{F}^{3+} \text { ) ONLY. All other components can be } \\
\text { gradually accrued to enhance efficiency of } \\
\text { yield/rates. High affinity recognitions are not } \\
\text { necessary to start off with. (The components } \\
\text { above are also required for ETC-CRAS } \\
\text { proposal! However, the murburn mechanism is } \\
\text { not based in any "intelligent gambit logic" of } \\
\text { using a bait to lure the prey. That is- a proton } \\
\text { pump based TMP is not used and harnessed to } \\
\text { make ATP by a catalyst evolved to break it.) }\end{array}$ & $\begin{array}{l}\text { A bevy of electron donor and acceptor } \\
\text { proteins/molecules embedded in a closed } \\
\text { phospholipid membrane, which is in turn } \\
\text { enclosed in yet another membrane + } \\
\text { electron transfer catalysts working in } \\
\text { intricately woven deterministic pathways } \\
\text { recognizing each other with topological } \\
\text { affinity + proteins that can couple } \\
\text { electron transfer to trans-membrane } \\
\text { proton pumping and TMP generation + } \\
\text { 'high concentration of protons in' to } \\
\text { serve proton pumps AND the exclusive } \\
\text { option of 'low protons in' for generating } \\
\text { TMP + molecular motors triggered by } \\
\text { protons/TMP, etc. and more...... } \\
\text { Definitely, irreducibly complex! }\end{array}$ \\
\hline 2 & $\begin{array}{l}\text { Is the } \\
\text { mechanism } \\
\text { relevant } \\
\text { across other } \\
\text { redox } \\
\text { metabolic } \\
\text { systems? }\end{array}$ & $\begin{array}{l}\text { Explains reaction kinetics and mechanism of } \\
\text { heme/flavin proteins, reasons fundamental } \\
\text { physiologies of maverick dose responses, } \\
\text { thermogenesis and xenobiotic metabolism in the } \\
\text { cytoplasmic microsomal system, thermogenesis } \\
\text { with uncoupling proteins, } \\
\text { photophosphorylation, etc. Therefore, the } \\
\text { reaction logic is ubiquitous. }\end{array}$ & $\begin{array}{l}\text { Since TMP is not involved in } \\
\text { microsomal systems, its mechanistic } \\
\text { principles of uncoupling are false. } \\
\text { Dissipation of TMP cannot be a viable } \\
\text { reason for thermogenesis in normal or } \\
\text { adipose tissues. The mechanistic } \\
\text { terminology/concept is only heard of } \\
\text { within the realm of bioenergetics. }\end{array}$ \\
\hline
\end{tabular}

